\title{
BMJ Open Protocol for a pre-implementation and post-implementation study on shared decision-making in the surgical treatment of women with early-stage breast cancer
}

\author{
Wilma Savelberg, ${ }^{1}$ Albine Moser, ${ }^{2}$ Marjolein Smidt, ${ }^{1}$ Liesbeth Boersma, ${ }^{3}$ \\ Christel Haekens, ${ }^{1}$ Trudy van der Weijden ${ }^{4,5}$
}

To cite: Savelberg W, Moser A, Smidt M, et al. Protocol for a preimplementation and postimplementation study on shared decision-making in the surgical treatment of women with early-stage breast cancer. BMJ Open 2015;5:e007698.

doi:10.1136/bmjopen-2015007698

- Prepublication history for this paper is available online. To view these files please visit the journal online (http://dx.doi.org/10.1136/ bmjopen-2015-007698).

Received 16 January 2015 Revised 19 February 2015 Accepted 26 February 2015

CrossMark

For numbered affiliations see end of article.

Correspondence to Dr Wilma Savelberg; w.savelberg@mumc.n

\section{ABSTRACT}

Background: The majority of patients diagnosed with early-stage breast cancer are in a position to choose between having a mastectomy or lumpectomy with radiation therapy (breast-conserving therapy). Since the long-term survival rates for mastectomy and for lumpectomy with radiation therapy are comparable, patients' informed preferences are important for decision-making. Although most clinicians believe that they do include patients in the decision-making process, the information that women with breast cancer receive regarding the surgical options is often rather subjective, and does not invite patients to express their preferences. Shared decision-making (SDM) is meant to help patients clarify their preferences, resulting in greater satisfaction with their final choice. Patient decision aids can be very supportive in SDM. We present the protocol of a study to $\beta$ test a patient decision aid and optimise strategies for the implementation of SDM regarding the treatment of early-stage breast cancer in the actual clinical setting.

Methods/design: This paper concerns a preimplementation and post-implementation study, lasting from October 2014 to June 2015. The intervention consists of implementing SDM using a patient decision aid. The intervention will be evaluated using qualitative and quantitative measures, acquired prior to, during and after the implementation of SDM. Outcome measures are knowledge about treatment, perceived SDM and decisional conflict. We will also conduct face-to-face interviews with a sample of these patients and their care providers, to assess their experiences with the implementation of SDM and the patient decision aid.

Ethics and dissemination: This protocol was approved by the Maastricht University Medical Centre (MUMC) ethics committee. The findings will be disseminated through peer-reviewed journal articles and presentations at national conferences. Findings will be used to finalise a multi-faceted implementation strategy to test the implementation of SDM and a patient decision aid in terms of cost-effectiveness, in a multicentre cluster randomised controlled trial (RCT). Study registration number: NTR4879.

\section{INTRODUCTION}

Several studies have revealed that mastectomy and breast-conserving surgery with radiation therapy are comparable in terms of local control and long-term survival. ${ }^{1}$ In addition, some studies found no difference in quality of life between patients treated with breast-conserving treatment or mastectomy, while other studies reported higher quality of life after breast-conserving treatment compared with mastectomy. ${ }^{2}$ Many patients with early-stage breast cancer face the dilemma of choosing between these two options when considering breast surgery. Deciding between these two can be regarded as being influenced by patient preferences.

Evidence is growing that patient preferences may vary substantially between individuals. ${ }^{3}$ In addition to survival, important factors in the decision-making process are the patient's age, family history and preference for reconstruction and quality of life. At the time of diagnosis, patients with breast cancer have their own values, concerns and knowledge, which can influence their treatment preferences. ${ }^{2}$

There is also increasing evidence that most patients want to be involved in treatment decisions. ${ }^{4}$ For many patients, greater involvement in cancer treatment decisions can improve their knowledge about treatment benefits, enhance their satisfaction with the decision and improve their quality of life. ${ }^{5}{ }^{6}$ It is important to present the information to patients as neutrally as possible and to involve them in the decision, in order to achieve a tailor-made, personalised treatment plan. Shared decision-making (SDM) is regarded as a promising model to achieve such patient involvement. SDM has been 
defined as: "an approach where clinicians and patients share the best available evidence when faced with the task of making decisions, and where patients are supported to consider options, to achieve informed preferences". 7

Nevertheless, there is a problem with the implementation of SDM in clinical practice. Physicians typically feel they do not have the time, or lack the skills, to offer a complete and balanced presentation of the pros and cons of suitable medical options. ${ }^{8}$ One measure to support SDM would be to use a patient decision aid, ${ }^{9-11}$ which provides information facilitating discussion and deliberation about treatment options. There is strong evidence that these aids are effective in achieving informed preferences and decisions that are more in line with patient preferences. ${ }^{4}$ The distribution and use of patient decision aids is also associated with increased knowledge about options and decreased decisional conflict. ${ }^{10}$

We feel that integrating a patient decision aid in the daily workflow at the clinic is the first move towards implementing more uniform and objective SDM in an actual oncology setting. In the USA and the UK, patient decision aids have been implemented as part of a usual care programme in breast cancer centres. Both programmes consist of various decision aid materials, including videos and booklets. In addition, both programmes provide tools involving question listing, audiorecording and note-taking services by trained associates, who are either premedical interns or professional counsellors. ${ }^{6} 12 \quad 13$ Although these are excellent initiatives, one should be cautious about merely copying these approaches, as implementation should take political, cultural and economic conditions into consideration. ${ }^{14} 15$

The challenge is to feasibly embed SDM and the patient decision aid in the clinical practice workflow. It is well known that the implementation of new methods in clinical practice can be difficult, as clinicians have busy schedules filled with daily routines and there is often no obvious motivation nor time to change them. ${ }^{16}$ Implementation strategies which do not focus on the problems that health professionals' experiences are less effective in accomplishing change. ${ }^{1}$

One single intervention is probably insufficient to achieve successful implementation of SDM using patient decision aids in clinical practice, so a systematic approach and careful planning of implementation activities is needed. ${ }^{15}$ Achieving successful implementation requires devoting attention to the process of developing the patient decision aid, its scientific basis, its format and its content. The implementation of change model describes the involvement of different target groups in the 'development, testing and execution of an implementation'. ${ }^{15}$

\section{OBJECTIVES AND RESEARCH QUESTIONS}

The objectives of this project are to pilot-test and optimise strategies for the implementation of SDM for patients with early-stage breast cancer in an actual clinical setting.

Our hypothesis is that a multifaceted strategy would enable us to implement SDM in such a way that it meets the needs and demands of professionals and patients, without disrupting daily practice.

Primary research questions to support the development of the implementation strategy are:

1. What are the perceived barriers and facilitators, needs and preferences of patients and the professionals of the breast cancer team with regard to:

- The integration of the patient decision aid in patient care, making it acceptable for integration in the clinical workflow;

- The model of SDM, that is, how, when and by whom it should be integrated into the clinical pathway;

- Coaching of the professionals or instructions for SDM?

Secondary research questions to support the design of a large-scale study to evaluate the final implementation strategy are:

1. What is the impact of the implementation of the patient decision aid on the process of SDM, on the patients' knowledge and on decisional conflict?

2. To what extent does the decision aid produce changes in the intended (ie, preferences) and final treatment decisions by doctors and patients?

\section{METHOD}

\section{General design}

The design of the study is the pre-implementation and post-implementation to $\beta$ test the patient decision aid and develop related implementation strategies in the clinic, involving quantitative and qualitative methods. For the sake of readability, the methods are described below in the sequence in which they are carried out, starting with the quantitative data collection (which is not in line with the above sequence of the research questions).

In the preimplementation period ( 3 months), data are collected from patients with early-breast cancer $(\mathrm{N}=40)$ receiving care as usual. During the implementation period (5 months), data are collected from women $(\mathrm{N}=40)$ taking part in the process of SDM using the patient decision aid. This study last from 1 October 2014 to 1 June 2015.

\section{Setting}

Data collection takes place in four Dutch hospitals in the western, central and southern parts of the Netherlands.

\section{Participants}

\section{Patients}

Each hospital will include 10 patients with newly diagnosed early-breast cancer (stage I or II), who are eligible for breast-conserving therapy or mastectomy as their 
primary therapy during the preintervention period. During the intervention period, each hospital will again include 10 patients (mother sample) with newly diagnosed early-breast cancer (stage I or II), who are eligible for breast-conserving therapy or mastectomy as their primary therapy. From this sample, 4 patients of each hospital will be included to take part in the qualitative study during the postintervention period (table 1).

\section{Inclusion criteria}

The study will include patients diagnosed with stage I or II breast cancer, provided the two treatment options, mastectomy or breast-conserving surgery with radiotherapy, are applicable. Eligible patient should be able to speak and understand Dutch.

Eligible patients will be identified at the multidisciplinary meetings of the breast cancer oncology team. The surgeon or nurse revealing the cancer diagnosis to the patients will inform them about this study. They will also provide the patients with an information letter to inform them about the aim and procedure of the study, and the importance of their participation: the letter includes an informed consent form.

Handling of personal data will be in accordance with the Dutch Personal Data Protection Act and Medical Research (Human Subjects) Act.

\section{Professionals}

In each hospital, all breast surgeons, radiation oncologists, nurse practitioners and nurses taking part in the education and decision-making process will be invited to participate in the intervention.

\section{Intervention}

The intervention consists of instruments and activities to implement SDM, including the patient decision aid, in the clinic.

A draft patient decision aid has been developed by a research team from Maastricht University Medical Centre (MUMC) and the Amsterdam Academic Medical Centre (AMC), based on existing patient decision aids (http://www.kiesbeter.nl), the Dutch clinical practice guidelines on breast cancer, additional literature and expert opinion. This draft was $\alpha$ tested in a first trial round for professionals and patients. The decision aid is expected to be an easy-to-use website, which is made available to patients included in the study through a link and a password. It offers an explanation of the surgical options, as well as a brief overview of considerations that could be relevant to women regarding their own values, preferences and concerns, and enables patients to navigate through the decision aid. Comprehensive information is presented about mastectomy and breastconserving surgery, including numerical information about survival and recurrence rates, pros and cons of treatments and the side effects. The verbal information is supported by pictures and graphs. Finally, the information is summarised in a factsheet. The decision aid further includes a number of questions to help women identify their values. ${ }^{17}$ To make the information more accessible during the consultation, we have developed an additional options grid, in the form of a one-page table summarising the treatment options, which can also be used at home.

The patient decision aid will be made available to clinicians in February 2015. Participating clinicians need to learn to use the patient decision aid correctly. To achieve this, they will be instructed regarding SDM and its favourable effects. The instructions include a compact e-learning component with role modelling and suggestions for integrating the patient decision aid in the clinical pathway and for task delegation. This will be provided in a tailored manner, with a certain degree of local adaptation allowed for each hospital and department.

\section{Data collection and analysis}

Quantitative data (see below) will be collected from 10 patients of each hospital during the pre-implementation period (1 November 2014-1 February 2015) and from 10 patients of each hospital during the intervention period (1 February 2015-1 May 2015), using consecutive

\begin{tabular}{|c|c|c|c|c|c|}
\hline \multicolumn{2}{|c|}{ Preintervention } & \multicolumn{2}{|c|}{ Intervention } & \multicolumn{2}{|c|}{ Postintervention } \\
\hline \multicolumn{2}{|c|}{$\overline{\text { Quantitative data collection }}$} & \multicolumn{2}{|c|}{$\overline{\text { Quantitative data collection }}$} & \multicolumn{2}{|c|}{ Qualitative data collection } \\
\hline Hospital & Patients (n) & Hospital & Patients (n) & Hospital & Interviews (n) \\
\hline \multicolumn{6}{|l|}{ Patients } \\
\hline $\mathrm{H} 1$ & 10 & $\mathrm{H} 1$ & 10 & $\mathrm{H} 1$ & 4 of the 10 \\
\hline $\mathrm{H} 2$ & 10 & $\mathrm{H} 2$ & 10 & $\mathrm{H} 2$ & 4 of the 10 \\
\hline $\mathrm{H} 3$ & 10 & H3 & 10 & $\mathrm{H} 3$ & 4 of the 10 \\
\hline $\mathrm{H} 4$ & 10 & $\mathrm{H} 4$ & 10 & $\mathrm{H} 4$ & 4 of the 10 \\
\hline \multicolumn{6}{|c|}{ Professionals } \\
\hline & & & & $\mathrm{H} 1$ & All participating professionals, \\
\hline & & & & $\mathrm{H} 2$ & but at least 1 surgeon and \\
\hline & & & & $\mathrm{H} 3$ & nurse per hospital \\
\hline & & & & $\mathrm{H} 4$ & \\
\hline
\end{tabular}


sampling of patients who fill in the questionnaires. In addition, 4 of the 10 patients included during the intervention period will be recruited by surgeons and nurses for collection of qualitative data (see below) from 1 May 2015 to 1 June 2015 , that is, after the intervention. These patients will be selected using convenience sampling.

The results from both study groups will be compared by descriptive statistical procedures to identify differences between the two groups.

\section{QUANTITATIVE DATA}

\section{Variables and instruments}

A variety of instruments will be used to assess different outcome measures (table 2):

- Patients' knowledge about breast cancer and treatment options will be assessed using an adapted breast cancer information test. ${ }^{18}$ This scale includes knowledge-type questions about early-stage breast cancer treatment. Questions are answered using true or false.

- Decisional conflict will be assessed using the Decisional Conflict Scale (DCS). ${ }^{19}$ This 16 -item scale captures factors associated with decisional conflict or uncertainty. The DCS has three subscales: decisional uncertainty, factors that contribute to uncertainty and perceived effective decision. Each of these items is scored on a five-point Likert scale from 1 (strongly agree) to 5 (strongly disagree).

- The process of SDM will be assessed by the perceptions of patients and clinicians, using the SDM-Q9 instrument. ${ }^{20}$ The Dutch version of the SDM-Q9 has recently been validated. The instrument has a dyadic approach and consists of nine statements, which can be rated on a six-point scale from 0 (completely disagree) to 5 (completely agree). Summing all items leads to a raw total score between 0 and 45 .

- The process of SDM will also be assessed by an independent observer from audiorecordings of the consultations. The audiotapes will be rated with the Observer OPTION (Observing patient involvement) 12 item by two researchers. ${ }^{21}$ The Observer OPTION consists of a set of competences, including problem definition, explaining legitimate choices, portraying options and communication risk, and conducting the decision process. The instrument aims to measure to what extent the patient is involved in the decision about the treatment, and consists of 12 items. The measurement level is ordinal with scores of $0-4$.

- An audit will be conducted on the actual decision taken.

- The time that participants spend reading the patient decision aid will be recorded.

- The time spent by the professionals on consultation will be determined from the audiorecordings.

\section{QUALITATIVE DATA}

\section{Variables and instruments}

Information on the patient decision aid will be obtained from patients by means of semistructured interviews which will be conducted during the postintervention period. ${ }^{22}$ An interview guide will be prepared for the patient interviews. The questions will focus on the content, presentation and navigation (user-friendliness)

\section{Table 2 Outcome measures}

\begin{tabular}{|c|c|c|c|c|c|}
\hline & \multicolumn{2}{|c|}{ Preintervention period } & \multicolumn{2}{|c|}{ Intervention period } & \multirow{3}{*}{$\begin{array}{l}\text { Postintervention period } \\
\text { May-June } 2015 \\
\text { Qualitative collection } \\
\text { among patients and } \\
\text { professionals }\end{array}$} \\
\hline & $\begin{array}{l}\text { November 2014- } \\
\text { January } 2015\end{array}$ & $\begin{array}{l}\text { February } \\
2015\end{array}$ & $\begin{array}{l}\text { February-May } \\
2015\end{array}$ & $\begin{array}{l}\text { June } \\
2015\end{array}$ & \\
\hline & $\begin{array}{l}\text { Quantitative } \\
\text { collection } \\
\text { among patients }\end{array}$ & & $\begin{array}{l}\text { Quantitative } \\
\text { collection } \\
\text { among patients }\end{array}$ & & \\
\hline \multicolumn{6}{|l|}{ Measures } \\
\hline $\begin{array}{l}\text { Time to read the patient decision } \\
\text { aid knowledge about breast } \\
\text { cancer and treatment (breast } \\
\text { cancer information test) }\end{array}$ & $x$ & & $\begin{array}{l}X \\
X\end{array}$ & & \\
\hline Decisional Conflict Scale (DCS) & & $\mathrm{X}$ & & $x$ & \\
\hline $\begin{array}{l}\text { Perceived shared } \\
\text { decision-making (SDM-Q9) }\end{array}$ & $x$ & & $x$ & & \\
\hline $\begin{array}{l}\text { Process of shared } \\
\text { decision-making objectivised } \\
\text { observing patient involvement } \\
\text { (OPTION) }\end{array}$ & $x$ & & $x$ & & \\
\hline Consultation time & $\mathrm{X}$ & & $\mathrm{X}$ & & \\
\hline Experiences & & & & & $x$ \\
\hline Perceptions of feasibility & & & & & $x$ \\
\hline Usability & & & & & $x$ \\
\hline Utility & & & & & $x$ \\
\hline Satisfaction & & & & & $x$ \\
\hline
\end{tabular}


and the perceived usefulness of the patient decision aid (utility) and on the patients' experiences with the SDM process.

Qualitative data from the clinicians will be obtained through focus group or face-to-face discussions to evaluate the SDM and the use of the decision aid, during the postintervention period. A question route $^{23}$ will be defined moving from general to more specific issues, focusing on their perceptions and experiences of applying SDM and the patient decision aid, as well as general appreciation and the intention to recommend the patient decision aid to colleagues. We will also discuss barriers and facilitators (positive features, changes needed, relevance, timing of use).

The interviews with patients and the discussions with the clinicians will be audiotaped and transcribed verbatim. Both data sets will be analysed using the constant comparison method. ${ }^{24}$

\section{DISCUSSION}

\section{Design}

The design combines the strengths of quantitative and qualitative research. ${ }^{25}$ The qualitative data obtained, which are based on human experience, will be examined in detail and in depth. The data will be used to assess the usability of the patient decision aid and the barriers and facilitators for the implementation of the decision aid in a small group, including professionals and patients. We aim to evaluate the feasibility of the measurement instruments and the potential effect of the implementation strategies (instructions and patient decision aid) on the performance and experience of SDM compared with a historical control group, to enable a power calculation for a large multicentre randomised controlled trial (RCT).

\section{Sample size}

Limitations of the sampling process in view of the available time, the size of the target group and the nature of the intervention, it is not possible to conduct a random sampling. During the course of the study, all patients with early-stage breast cancer in the participating hospitals will be invited to participate, until we reach the number of 10 participants for each hospital. Thus, over a certain period, the entire accessible population will be studied. ${ }^{26}$ Inviting all members of the accessible population reduces the risk of bias. According to Johanson and Brooks, ${ }^{27}$ a number of 30-40 patients is recommended for a pilot study whose purpose is a preliminary survey. The sample size in this study meets these recommendations.

Sample size for qualitative data collection in qualitative studies tends to be small. The number of participants needed depends on the point where data saturation is reached. Data saturation is expected to occur after 12 participants have been interviewed, provided these patients are not verbally vulnerable. ${ }^{25}$ Since little is known about SDM and the use of patient decision aids in the clinical setting with respect to this data, this study will include 20 patients who are exposed to the intervention. We thus expect that data saturation will occur and that the process will yield rich and in-depth findings.

\section{Implementation of the intervention}

Achieving success requires a systematic approach and careful planning of the implementation strategy. Joseph-Williams $e t a l^{28}$ argues that barriers could be overcome by behavioural changes at the level of the patient, clinicians/healthcare team and the organisation in daily care. In this study, we primarily focus on the clinicians/ healthcare team and the organisation, since interventions are successfully implemented when barriers regarding these factors are overcome. ${ }^{1}$

Worldwide training programmes on SDM vary greatly in what they offer and how they present it. In addition, evidence of their effectiveness is inconclusive. ${ }^{29}$ We have opted for e-learning because the purpose in this study is to examine the support and assistance required to develop suitable educational programmes in an RCT. E-learning might be a promising strategy to support the implementation of SDM and patient decision aids in actual clinical settings.

\section{Measures}

The SDM-Q9 is a recently developed instrument measuring the perceptions of the stakeholders of the SDM process. ${ }^{30}$ We decided to use this instrument because of the dyadic approach in SDM. Doctors and patients are acknowledged and seen as equally involved in the consultation and decision-making. A study concerning the use of the dyadic OPTION scale supports this dyadic approach. $^{31}$

The process of SDM will be analysed in this study by means of the OPTION scale. This scale has been validated and is based on the phases of SDM. In her review, Stacey found two studies using the OPTION scale to evaluate the interaction between patient and professional. Recently, the Observer OPTION 5 item was introduced as an instrument focusing on essential aspects of SDM, providing shorter measurements. Despite its promising results, it needs more empirical work ${ }^{32}$ to explore its scientific value. To the best of our knowledge, there are few studies combining all of these measurements to gain extensive insights into the process of SDM and the implementation of a patient decision aid.

We originally intended to measure decisional conflict immediately after the completion of surgical treatment. However, based on recommendations by the professionals, whose experience is that satisfaction or regret does not occur until later in the process, we decided to measure decisional conflict 3 months after the surgical treatment.

In conclusion, this study seeks to examine the main obstacles and success factors for the implementation of SDM using a patient decision aid, and to determine the most favourable way to integrate this in the clinical 
pathway. In addition, we will investigate the impact of this implementation on several outcome variables. These will be used as a basis to design a multifaceted complex implementation strategy, with the intention of testing the implementation of SDM and a patient decision aid in a multicentre cluster RCT.

\section{Author affiliations}

${ }^{1}$ Oncology Center, Maastricht University Medical Center, Maastricht, The Netherlands

${ }^{2}$ Zuyd University of Applied Sciences, Heerlen, The Netherlands

${ }^{3}$ Department of Radiotherapy, Maastricht University Medical Center, Maastricht, The Netherlands

${ }^{4}$ Department of Family Medicine, Maastricht University, Maastricht, The Netherlands

${ }^{5}$ School for Public Health and Primary Care (CAPHRI), Maastricht University, Maastricht, The Netherlands

Contributors WS, AM and TvdW developed the study protocol. TvdW obtained research funding. WS, AM, TvdW, LB, MS and $\mathrm{CH}$ participated in the final design of the study and drafting/revising of this manuscript. All authors read and approved the final manuscript.

Funding This work was supported by Pink Ribbon, grant number 2012.PS23. C152. Pink Ribbon has approved the project proposal in which the method and design of the study was determined. The study protocol was in accordance with the project proposal.

Competing interests None.

Patient consent Obtained

Ethics approval The study has been approved by the Maastricht University Medical Centre (MUMC) ethics committee.

Provenance and peer review Not commissioned; peer-reviewed for ethical and funding approval prior to submission.

Data sharing statement There are no additional unpublished data.

Open Access This is an Open Access article distributed in accordance with the Creative Commons Attribution Non Commercial (CC BY-NC 4.0) license, which permits others to distribute, remix, adapt, build upon this work noncommercially, and license their derivative works on different terms, provided the original work is properly cited and the use is non-commercial. See: http:// creativecommons.org/licenses/by-nc/4.0/

\section{REFERENCES}

1. Baker R, Camosso-Stefinovic J, Gillies C, et al. Tailored interventions to overcome identified barriers to change: effects on professional practice and health care outcomes. Cochrane Database Syst Rev 2010;(3):CD005470.

2. Fisher $\mathrm{B}$, Anderson $\mathrm{S}$, Bryant $\mathrm{J}$, et al. Twenty-year follow-up of a randomized trial comparing total mastectomy, lumpectomy, and lumpectomy plus irradiation for the treatment of invasive breast cancer. N Engl J Med 2002;347:1233-41.

3. Obeidat R, Finnell DS, Lally RM. Decision aids for surgical treatment of early stage breast cancer: a narrative review of the literature. Patient Educ Couns 2011;85:e311-21.

4. Stacey D, Bennett $C L$, Barry MJ, et al. Decision aids for people facing health treatment or screening decisions. Cochrane Database Syst Rev 2011;(10):CD001431.

5. Livaudais JC, Franco R, Fei K, et al. Breast cancer treatment decision-making: are we asking too much of patients? J Gen Intern Med 2013;28:630-6.
6. Belkora JK, Loth MK, Volz S, et al. Implementing decision and communication aids to facilitate patient-centered care in breast cancer: a case study. Patient Educ Couns 2009;77:360-8.

7. Elwyn G, Frosch D, Thomson $\mathrm{R}$, et al. Shared decision making: a model for clinical practice. J Gen Intern Med 2012;27:1361-7.

8. Fowler FJ Jr, Levin CA, Sepucha KR. Informing and involving patients to improve the quality of medical decisions. Health Aff (Millwood) 2011;30:699-706.

9. Stacey D, Kryworuchko J, Bennett C, et al. Decision coaching to prepare patients for making health decisions: a systematic review of decision coaching in trials of patient decision AIDS. Med Decis Making 2012;32:E22-33.

10. Belkora JK, Volz S, Teng AE, et al. Impact of decision aids in a sustained implementation at a breast care center. Patient Educ Couns 2012;86:195-204.

11. Waljee JF, Rogers MA, Alderman AK. Decision aids and breast cancer: do they influence choice for surgery and knowledge of treatment options? J Clin Oncol 2007;25:1067-73.

12. Belkora JK, Teng A, Volz S, et al. Expanding the reach of decision and communication aids in a breast care center: a quality improvement study. Patient Educ Couns 2011;83:234-9.

13. Pass M, Belkora J, Moore D, et al. Patient and observer ratings of physician shared decision making behaviors in breast cancer consultations. Patient Educ Couns 2012;88:93-9.

14. Lloyd A, Joseph-Williams N, Edwards A, et al. Patchy 'coherence': using normalization process theory to evaluate a multi-faceted shared decision making implementation program (MAGIC). Implement Sci 2013;8:102.

15. Grol R, Wensing M, Eccles M, et al. Improving patient care: the implementation of change in health care. Wiley, 2013.

16. Elwyn G, Laitner S, Coulter A, et al. Implementing shared decision making in the NHS. BMJ 2010;341:c5146.

17. Fagerline $\mathrm{A}$, Pignone $\mathrm{M}$, Abhyankar $\mathrm{P}$, et al. Clarifying values: an updated review. BMC Med Inform Decis Mak 2013;13(Suppl 2):S8.

18. Sawka CA, Goel V, Mahut CA, et al. Development of a patient decision aid for choice of surgical treatment for breast cancer. Health Expect 1998:1:23-36.

19. Stalmeier PF, Roosmalen MS, Verhoef LC, et al. The decision evaluation scales. Patient Educ Couns 2005;57:286-93.

20. Kriston L, Scholl I, Hölzel L, et al. The 9-item Shared Decision Making Questionnaire (SDM-Q-9). Development and psychometric properties in a primary care sample. Patient Educ Couns 2010;80:94-9.

21. Elwyn G, Edwards $A$, Wensing $M$, et al. Shared decision making developing the OPTION scale for measuring patient involvement. Qual Saf Health Care 2003;12:93-9.

22. Kvale S, Brinkmann S. Interviews. Learning the craft of qualitative research interviewing. London: Sage, 2009.

23. Krueger R, Casey M. Focus groups. A practical guide for applied research. 4th edn. Thousand Oaks, CA: Sage.

24. Corbin J, Strauss A. Basics of qualitative research. 3rd edn. Thousand Oaks, CA: Sage, 2006.

25. O'Connor A, Llewellyn-Thomas H, Stacey D. IPDAS Collaboration Background Document. 2005.

26. Polit D, Beck C. Nursing reasearch; generating and assessing evidence for nursing practice. 9th edn. Wolters kluwer Health, 2012

27. Johanson GA, Brooks GP. Initial scale development: sample size for pilot studies. Educ Psychol Measures 2010;70:394-400.

28. Joseph-Williams NG, Elwyn G, Edwards A. Knowledge is not power for patients: a systematic review and thematic synthesis of patient-reported barriers and facilitators to shared decision making Patient Educ Couns 2013;94:291-309.

29. Legare F, Politi MC, Drolet R, et al. Training health professionals in shared decision-making: an international environmental scan. Patient Educ Couns 2012;88:159-69.

30. Scholl I, Kriston L, Dirmaier J, et al. Comparing the nine-item shared decision-making questionnaire to the OPTION Scale-an attempt to establish convergent validity. Health Expect 2015;18:137-50.

31. Melbourne E, Roberts S, Durand MA et al. Dyadic OPTION: measuring perceptions of shared decision-making in practice. Patient Educ Couns 2011;83:55-7.

32. Elwyn G, Lloyd A, Joseph-Williams N, et al. Option grids: shared decision making made easier. Patient Educ Couns 2013;90:207-12. 